\title{
Paradojas de la participación juvenil y desafíos de la educación ciudadana en Chile
}

The Paradoxes of Juvenile Participation and the Challenges of Civic Education in Chile Paradoxes de la participation juvénile et défis de l'éducation citoyenne au Chili Paradoxos entre a participação juvenil e os desafios da educação para a cidadania no Chile

Fecha de recepción: 9 DE MARZO DE 2012/ Fecha de aceptación: 5 DE OCTUBRE DE 2013

Encuentre este artículo en http://magisinvestigacioneducacion.javeriana.edu.co/ doi:10.11144/Javeriana.M6-13.PPJD

Escrito por Luis Manuel Flores-GonzÁlez Pontificia Universidad Católica de Chile Santiago de Chile, Chile Imflores@uc.cl

Carolina Andrea García-González Pontificia Universidad Católica de Chile Santiago de Chile, Chile cagarcig@uc.cl

\section{Resumen}

El sistema democrático está enfrentando una profunda crisis de representatividad, cuya expresión máxima se encuentra en las generaciones más jóvenes. El objetivo de este artículo es reflexionar acerca de la vinculación de esta crisis y la paradoja sobre la que se funda el sistema democrático chileno, a partir del análisis de las contradicciones entre el discurso y la práctica de la participación juvenil, de las características de la educación ciudadana en Chile, encargada de formar a los ciudadanos que el sistema democrático necesita y del potencial efecto de la escuela en la formación de una ciudadanía democrática.

\section{Palabras clave autor}

Educación ciudadana, cohesión social, ciudadanía juvenil, paradojas de la democracia.

\section{Palabras clave descriptor}

Educación cívica, adultos jóvenes, participación política, democracia.

\section{Transferencia a la práctica}

La reflexión que se plantea en este artículo busca revelar las relaciones entre las paradojas del sistema democrático y la participación juvenil, con la educación ciudadana que se imparte en las escuelas chilenas, con la finalidad de que se traduzca en un aporte para evaluar las políticas públicas sobre educación ciudadana implementadas y para diseñar nuevas estrategias curriculares y pedagógicas que fortalezcan la tarea de formación ciudadana que se le ha encomendado a la escuela y así contribuir a la superación de la crisis que experimenta el sistema democrático.

Para citar este artículo / To cite this article / Pour citer cet article / Para citar este artigo

Flores-González, L. M. \& García-González, C. A. (2014). Paradojas de la participación juvenil y desafíos de la educación ciudadana en chile. magis, Revista Internacional de Investigación en Educación, 6 (13), 31-48. 


\section{Key words author}

Civic Education, Social Cohesion, Juvenile Citizens, Paradoxes of Democracy.

\section{Key words plus}

Civics, Young Adults, Political

Participation, Democracy.

\section{Mots clés auteur}

Education citoyenne, cohésion sociale citoyenneté juvénile, paradoxes de la démocratie.

\section{Mots clés descripteur}

Instruction civique, jeunes adultes, participation politique, démocratie.

\section{Abstract}

Democracy is facing a profound crisis in its capacity of representation, most manifest in the younger generations. This paper aims to discuss the link between this crisis and the paradox on which democracy is based in Chile. It analyses the contradictions between discourse and practice in juvenile participation, the features of civic education in Chile - which is entrusted with the education of the citizens needed in a democratic system-, and the potential effect of the school in the education of democratic citizens.

\section{Transference to practice}

The discussion set out in this article seeks to show the link between the paradoxes of democracy, juvenile participation and civic education practiced in Chilean schools. The aim is to contribute to the assessment of public policy on civic education and to the design of new pedagogical and curricular strategies that strengthen civic education, which has been entrusted to schools, so that the current crisis of the democratic system can be overcome.

\section{Résumé}

Le système démocratique est en train d'affronter une profonde crise de représentativité, dont la plus forte preuve se trouve dans les générations les plus jeunes. L'objectif de cet article est donc celui de faire une réflexion par rapport au lien entre cette crise et le paradoxe sur lequel est fondé le système démocratique chilienne, à partir de l'analyse des contradictions entre la parole et la pratique de la participation juvénile, des caractéristiques de l'éducation citoyenne au Chili, qui est chargée de la formation des citoyennes dont le système démocratique a besoin et du potentiel effet de l'école dans la formation d'une citoyenneté démocratique.

\section{Transfert à la pratique}

La réflexion qu'on expose dans cet article cherche montrer les rapports entre les paradoxes du système démocratique et la participation juvénile, avec l'éducation citoyenne qui est accordée dans les écoles chiliennes, avec le but de contribuer à évaluer les politiques publiques par rapport à l'éducation citoyenne implémentée et pour dessiner des nouvelles stratégies curriculaires et pédagogiques pour renforcer la tâche de la formation citoyenne dont l'école est responsable et ainsi contribuer à surpasser la crise qui expérimente le système démocratique.

\section{Palavras-chave autor}

Educação cidadã, coesão social, cidadania juvenil, paradoxos da democracia.

\section{Palavras-chave descritor}

Educação cívica, adultos jovens, participação dos jovens, democracia.

\section{Resumo}

O sistema democrático está enfrentando uma profunda crise de representatividade e sua maior expressão se encontra nas gerações mais jovens. O objetivo deste artigo é refletir sobre a vinculação desta crise e o paradoxo sobre o qual se fundamenta o sistema democrático chileno, a partir da análise das contradições entre o discurso e a prática da participação dos jovens, das características da educação para a cidadania no Chile encarregada de educar cidadãos que o sistema democrático necessita e do potencial impacto da educação da escola para a cidadania democrática.

\section{Transferência à prática}

A reflexão proposta por este artigo procura revelar as relações entre os paradoxos do sistema democrático e a participação juvenil com a educação para a cidadania que se oferece nas escolas chilenas, a fim de contribuir com a avaliação das políticas de educação para a cidadania implementadas e desenhar novas estratégias de fortalecimento da tarefa da educação para a cidadania confiada à escola e contribuir para a superação da crise vivida pelo sistema democrático. 


\section{Introducción}

El sistema democrático chileno está experimentando una profunda crisis de representatividad. Si bien la institucionalidad sigue en pie, es decir, los cambios de Gobierno se desarrollan pacíficamente, las elecciones se realizan de forma periódica y las instituciones políticas cumplen con las funciones para las que fueron creadas, la indiferencia hacia la dimensión político-electoral comienza a profundizarse, sobre todo entre las generaciones más jóvenes.

Esta indiferencia parece estar en consecuencia con lo que algunos autores denominaron en su momento la "era del vacío" (Lipovetsky, 1986). Según esta interpretación, la sociedad occidental en su conjunto atraviesa un quiebre de sentido social y político, en relación con la exacerbación del individualismo contemporáneo que termina por negar la relación con el otro'. En esta medida, la ruptura es radical, porque es la subjetividad del otro y de sí mismo la que aparece fracturada y, en cierta forma, nihilizada.

La metáfora de Nietzsche sobre el desierto que avanza es retomada por Lipovetsky (1986) para indicar que el auténtico carácter trágico de la muerte de Dios que profetizara Nietzsche es la indiferencia y la naturalización de la falta de metarrelatos. A la actual sociedad posmoderna parece no importarle esta pérdida de sentido radical de la existencia. La verdadera tragedia no es que "Dios haya muerto", sino la situación de que su ausencia y vacío ya no son un drama para nadie:

La indiferencia crece. En ninguna parte el fenómeno es tan visible como en la enseñanza donde en algunos años, con la velocidad del rayo, el prestigio y la autoridad del cuerpo docente prácticamente han desaparecido [...]. Por eso, el colegio se parece más a un desierto que a un cuartel (y eso que un cuartel es ya en sí un desierto), donde los jóvenes vegetan sin grandes motivaciones ni intereses (p. 35).

Ahora bien, esta interpretación no niega de ninguna forma la existencia de focos de resistencia de los propios movimientos sociales a esta misma indiferencia que el sistema social parece alentar y reproducir. Cuando se dice que la apatía avanza, se quiere destacar que la idea de quiebre de sentido de común-unidad precede las justas reivindicaciones regionales que demandan algunos grupos e incluso países, por ejemplo, por mayor democracia.

La era del vacío es la era del no-sujeto, donde la "hiperprivatización" de la esfera pública hace desaparecer la experiencia del "nosotros", desde un concepto de tiempo solo como medida productiva y de consumo que termina por transformar la existencia humana en un anonimato sin rostro, sin ideales comunes y sin utopías como sociedad.

1 El hiperindividualismo no es, en rigor, una categoría sociológica; es más bien una interpretación de la sociedad moderna que indaga acerca de la hipótesis desde la cual la segmentación y atomización del sujeto es de carácter existencial más que de clase social. La era del vacío, en el contexto de Lipovetsky, corresponde al diagnóstico que postula no solo una crisis de la sociedad en su estructura económica — sean estos capitalistas o neoliberales-, sino a una época en que, como ya lo profetizara Nietzsche, el desierto avanza. El nihilismo no es la causa de la crisis postmoderna, sino una de sus consecuencias más trágicas en el sentido de que la muerte de Dios - o, como diría F. Lyotard, el fin de los metarrelatos - ya a nadie le importa.

En síntesis, el hiperindividualismo es una acusación respecto a una idea de sujeto entendido como realidad segmentada y, por ende, separado de sí mismo. El hiperindividualismo, en esta interpretación, corresponde a una crisis de la subjetividad en su estructura fundamental. El sujeto literalmente se desvanece sino es para y desde los otros (Lipovetsky, 1986).
Descripción del artículo | Article description | Description de I'article | Artigo descrição

Este artículo forma parte del Proyecto Fondecyt No. 1100649, "Vinculaciones entre la construcción y deconstrucción de la confianza y la cohesión social, en jóvenes estudiantes de educación secundaria: lineamientos para fortalecer la democracia" (Baeza, 2010-2012). 


\section{Planteamiento del problema}

Esta situación no es exclusiva de Chile. El estudio del PNUD (2004) que aborda la democracia en América Latina muestra la debilidad del compromiso democrático de la ciudadanía de la región. De los jóvenes de entre 16 y 29 años que participaron en este estudio, tan solo un $40 \%$ tiene orientaciones y opiniones democráticas claras, mientras que un $31,2 \%$ se muestra ambivalente frente al sistema democrático y $28,7 \%$ tiene orientaciones no democráticas. Aún más decisivo es que $56 \%$ de los entrevistados opina que el desarrollo económico es más importante que la democracia y $55 \%$ apoyaría a un Gobierno autoritario si pudiese solucionar los problemas económicos.

Estas cifras resultan un tanto paradójicas, cuando se considera que Chile y varios países latinoamericanos restablecieron la democracia solo hace un par de décadas, después de experimentar extensos Gobiernos autoritarios, caracterizados por la marginación política y por violaciones sistemáticas a los derechos humanos. Estos países cuentan en la actualidad con sistemas democráticos de hecho y de Derecho y con políticas de formación ciudadana implementadas mediante los sistemas educativos, orientados a desarrollar el compromiso y la conciencia de vivir en una sociedad democrática. Entre las explicaciones propuestas para comprender esta situación, se encuentra la estructura que han adquirido los sistemas democráticos que tienen un discurso participativo, pero en la práctica restringen los espacios de participación política o permiten que sean absorbidos por la lógica economicista del mercado y producen la mercantilización de todas las esferas sociales.

En Chile, durante los años de la dictadura², tanto la democracia como los espacios de participación ciudadana desaparecieron y se transformaron en un objetivo, bajo el supuesto de que, en democracia, las necesidades y opiniones de la ciudadanía serían escuchadas y atendidas, a diferencia de lo que ocurría bajo el régimen autoritario. La democracia pasó a concebirse como un destino unívoco, como la única forma que podía tomar la política para dar cabida a la autodeterminación colectiva suprimida por la dictadura (Lechner, 2002). Para alcanzar esta meta, la ciudadanía se organizó y luchó por recuperar los espacios de participación. La oposición política a la dictadura logró generar un movimiento social de carácter nacional para terminar con el régimen autoritario y volver a la democracia por medio de la opción "No" en el plebiscito de 1988. Sin embargo, contrario a lo que se esperaba, una vez recuperada la democracia, los procesos de participación que surgieron a fines de la dictadura fueron suprimidos y no se volvieron a impulsar (Otano, 1995).

El retorno a la democracia no cumplió con las expectativas. La transición tuvo un desarrollo pactado a puertas cerradas entre cúpulas políticas y el movimiento social, tan importante en la resistencia y oposición a la dictadura, no tuvo cabida. El futuro de Chile se decidió por la élite política, que parecía haber hecho suya la divisa del despotismo ilustrado: "una transición para el pueblo, pero sin el pueblo" (García, 2006, p. 472). Como consecuencia, la democracia alcanzada comenzó a perder su sentido, porque no era

2 El 11 de septiembre de 1973, las Fuerzas Armadas lideradas por el comandante en jefe del Ejército, Augusto Pinochet, derrocaron mediante un golpe del Estado al presidente democráticamente electo, Salvador Allende. Inició una dictadura que se extendió durante diecisiete años y se caracterizó por el cierre de todos los espacios de participación democrática, una política sistemática de represión y violación a los derechos humanos y la transformación de las estructuras políticas y económicas a partir de los principios neoliberales. Para profundizar en este tema, consultar Huneeus (2000); Cavallo y otros autores (2004). 
lo que se esperaba ni lo que se había prometido. Las preocupaciones cotidianas, los intereses y las opiniones de las personas no tuvieron el espacio anhelado. Pese al cambio de Gobierno, gran parte de la institucionalidad creada por el régimen militar se mantuvo y la participación ciudadana se vio cada vez más reducida a la participación electoral. La política se volvió un asunto de cúpulas de poder, lo que transformó a la democracia en una "oligarquía liberal", en un sistema en que el poder se vuelve un asunto privado, manejado por una minoría política que lo obtiene por vía electoral, pero sin dar más espacios de participación al ciudadano común y corriente (Castoriadis, 1986). Esta es una problemática transversal a todas las dimensiones sociales y, tal vez, es la educación con su seguidilla de reformas y ajustes que la han caracterizado desde la década del ochenta, uno de los mejores ejemplos de esta marginación (Cornejo, González \& Caldichoury, 2007; García Huidobro, 2006).

A la desilusión del sistema democrático se han sumado los efectos de la consolidación del sistema económico neoliberal. La lógica economicista ha invadido tanto la esfera pública como la esfera privada (Arendt, 2003), con la consecuente mercantilización de todos los aspectos de la vida cotidiana. Se ha impuesto una lógica en la que cada cosa, cada relación e incluso cada persona asume como medida y única razón de sí su valor de cambio en el mercado (Cornejo, González \& Caldichoury, 2007), lo que contribuye a profundizar la desigualdad y la segregación social. La educación es uno de los ejemplos más claros, ya que en todos sus niveles está imbuida por la competitividad, sin que esto haya mejorado su calidad. Por el contrario, se ha profundizado la segregación del sistema y se ha consolidado la relación entre resultados de aprendizaje y posición social.

El correlato de la mercantilización de la sociedad y de la disminución de los espacios de participación ha dado origen a una "democracia de baja intensidad" (Cornejo, González \& Caldichoury, 2007; Yarza, 2005), que limita la participación ciudadana a la esfera electoral y homogeniza la relación del ciudadano con el Estado y sus vinculaciones con el mercado. El Estado se ve reducido en sus funciones, se limita a propiciar las condiciones para que el sistema económico funcione y a establecer un orden social acorde con la mercantilización, lo que implica limitar la participación ciudadana a la capacidad de consumir. Por ello, todas las políticas públicas, en especial las educativas, son puestas al servicio de la lógica de mercado, para formar la subjetividad ciudadana que el modelo económico necesita: el consumidor competente, productivo, individualizado y apolítico.

En síntesis, el origen de la crisis del sistema democrático se encuentra en su paradoja fundacional.
La idea de tal paradoja no se limita a la idea corriente de una que ponga en entredicho el sentido común o muestre la contradicción de premisas lógicas que, al afirmar algo, se niegue aquello que se afirma. No es tampoco la paradoja de un argumento contradictorio ni falaz. La paradoja fundacional alude a una situación más radical que una simple oposición en los términos, puesto que es la misma contradicción la que instituye, en el despliegue de sí misma, una creencia fundamental o un horizonte de significado.

Los mitos fundadores, como lo estudia René Girard (1982), son ejemplos de cómo la negación, el crimen y los hechos de sangre instituyen un sentido originario que las sociedades y religiones replican desde una narración metahistórica. Esto es así en la medida en que son acontecimientos pertenecientes a un pasado primordial, que no tuvieron nunca un presente, pero que explican por antonomasia la regulación del tiempo presente de una comunidad determinada $y$, sobre todo, la perspectiva y los lineamentos del futuro. En analogía a la función originaria de las primeras narraciones de la historia, las paradojas fundacionales dotan de un sentido que, pese a corresponder a un conflicto originario-mítico o a una contradicción lógica, son vividas y asumidas por la comunidad como una forma de coherencia y de sentido. El mito fundacional de Chile no es distinto del resto del mundo americano: una tierra conquistada erigida sobre hechos de sangre. Así se origina desde un principio de dominación radical, con estructuras sociales sobre estructuras de legitimación del poder de unos pocos sobre otros desposeídos incluso del estado de naturaleza humana. Girard (2009) sostiene que, si bien en el origen los mitos fundadores de Occidente la violencia correspondía a los dioses, ahora la violencia se desplaza a las personas que la ejercen en nombre de aquellos (Girard, 2009).

De esta forma, la paradoja fundacional se refiere a una estructura asimétrica del poder, lo que supone también una visión asimétrica del otro como legítimo otro. La deslegitimación del otro estructura un sistema social en el que las diferencias sociales no son tanto la causa de la segmentación social, sino más bien su consecuencia más inmediata. La paradoja actual se esbozaría en los siguientes términos: pese a que el discurso político llama a la participación ciudadana y exige a las instituciones educativas la formación de un ciudadano crítico y participativo, el sistema funciona a partir de una democracia de baja intensidad. La democracia se reduce a la representatividad y limita la toma de decisiones políticas de los ciudadanos a la actividad de sufragar. Sin embargo, al mismo tiempo, entrega a los sujetos todas las herramientas para que se conviertan en simples consumidores económicamente activos, que gozan de libertad y poder aparentes para participar en el sistema económico y tomar decisiones que 
impactan en una noción de calidad de vida reducida a la adquisición de bienes de consumo.

El estudio de esta paradoja será desarrollada en este artículo en tres momentos. En primer lugar, se analizarán las paradojas de la ciudadanía juvenil, a partir de las contradicciones entre el discurso y la praxis juvenil. Aunque los datos de informes y estudios sobre la juventud muestran índices de apatía que evidenciarían que la cuestión política y social no parece incumbirles en absoluto, a lo largo de 2011 estos mismos jóvenes organizaron sofisticadas formas de resistencia social que, en muchos aspectos, tienen en jaque al sistema y a sus estructuras. En segundo lugar, se revisarán las características de la educación ciudadana en Chile y se relacionará el desfase entre la implementación curricular de las escuelas en el ámbito de la formación ciudadana con los límites de una política pública en educación, que parece más preocupada por los modelos de gestión de la enseñanza, que por los principios articuladores que deberían inspirarla. Por último, se examinará el efecto de la escuela en la formación ciudadana, al entregar antecedentes de la importancia de la instancia educativa y las implicaciones del desfase que se registra entre los objetivos curriculares de la formación ciudadana y las prácticas pedagógicas a partir de las cuales se implementa.

\section{Las paradojas de la ciudadanía juvenil en Chile}

La crisis de representatividad e incluso de legitimidad del sistema democrático ha tenido su máxima expresión en la actitud ciudadana de los jóvenes. En las últimas dos décadas se ha evidenciado una mayor disminución de su participación político-electoral (Riquelme, 1999). En las elecciones presidenciales del año 2009, los jóvenes de 18 a 29 años representaban un $17 \%$ del padrón electoral, ya que cerca de dos millones de ellos se marginaron del sistema (Servicio Electoral, 2010). La VI Encuesta Nacional de la Juventud (Injuv, 2010) muestra que solo un 20,8 \% de estos jóvenes estaba inscrito en los registros electorales (Gráfico 1). Entre las razones que argumentan para no inscribirse, priman aquellas opciones relacionadas con la falta de interés en la política $(34,7 \%)$, la creencia de que la política no soluciona los problemas (10,5\%), la falta de identificación con los partidos políticos existentes $(10,01 \%)$ y la falta de confianza en la clase política $(8,1 \%)$.

Gráfico 1.

Inscripción electoral

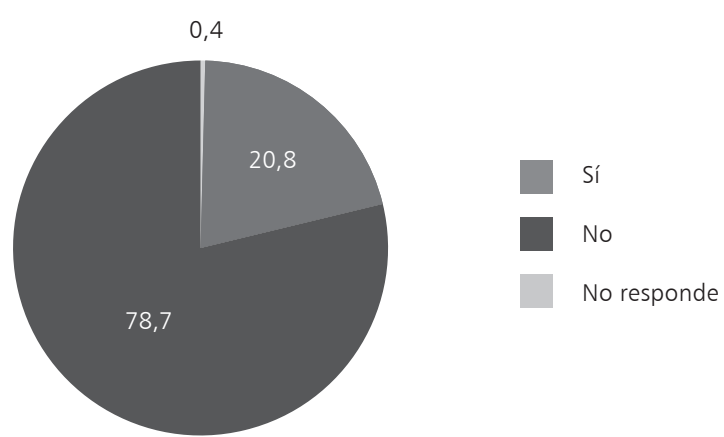


Esta misma encuesta evidencia que el Congreso y los partidos políticos son las organizaciones que más desconfianza generan en los jóvenes (Tabla 1), situación que es compartida por el resto de la sociedad (CERC, 2011), es decir, tanto los jóvenes como la ciudadanía general tienen baja confianza en instituciones básicas para el sistema democrático.

Tabla 1.

Confianza en las instituciones

\begin{tabular}{lcc}
\hline \multicolumn{1}{c}{ Institución } & Confianza (\%) & Desconfianza(\%) \\
\hline Partidos políticos & 2,2 & 60 \\
\hline Congreso & 2,5 & 48 \\
\hline Políticos & 2,8 & 54,5 \\
\hline Parlamentarios & 2,7 & 54 \\
\hline
\end{tabular}

Fuente: Injuv (2010)

La estabilidad del sistema democrático también se ve afectada por el bajo compromiso que los jóvenes tienen con él. Solo un $43 \%$ de los encuestados considera que la democracia es preferible frente a cualquier forma de gobierno, mientras que a un $28,9 \%$ le es indiferente el régimen de Gobierno y un 9,5\% considera que en algunas circunstancias es preferible un Gobierno autoritario.

Gráfico 2.

Posición frente a la democracia como forma de gobierno

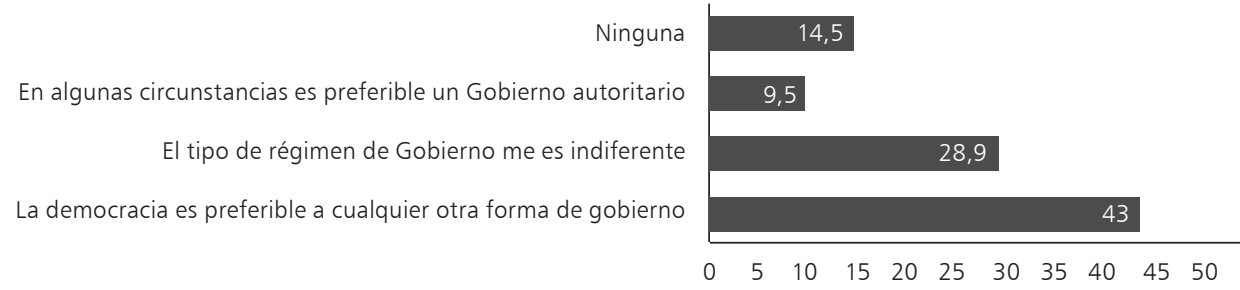

Fuente: Injuv (2010)

En relación con lo anterior, el grado de satisfacción con el sistema democrático también es bajo. Solo un $27,3 \%$ de los jóvenes encuestados se siente satisfecho, en tanto que un $39,1 \%$ no está satisfecho ni insatisfecho y un $24,1 \%$ se manifiesta definitivamente insatisfecho o muy insatisfecho.

Gráfico 3.

Satisfacción con la democracia

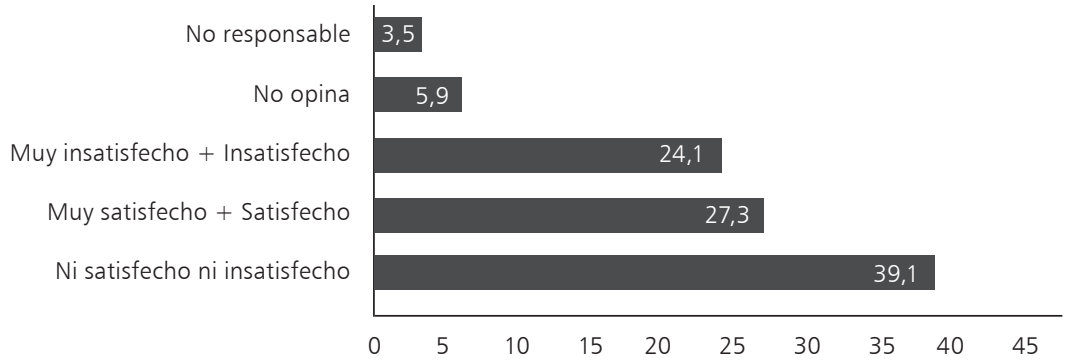

Fuente: Injuv (2010) 
En la misma encuesta se puede apreciar que los jóvenes valoran mucho la tolerancia y el respeto a la diversidad y manifiestan una activa participación en organizaciones sociales de carácter solidario y local o en subculturas urbanas. Durante mucho tiempo se asumió que esta participación era de carácter individualista y despolitizada (Cox, 2006), que hacía más referencia a un "yo" que ayuda a otros que están en problemas, que a un "nosotros". El quiebre del "nosotros" representa que el nudo del problema social de la participación desborda la disyuntiva entre inscribirse o no en los registros electorales y nos sitúa en la relación profunda que hay entre subjetividad y política.

Además de estudios cuantitativos que muestran la escasa confianza y participación de los jóvenes en el ámbito de la política y la participación ciudadana, encontramos trabajos muy relevantes desde el punto de vista cualitativo que muestran la misma tendencia, referida a la deslegitimación de la política por parte de los jóvenes (Baeza, 2010-2012).

Por otra parte, autores desde la Neurociencia (Varela, 2000) hasta la Política y la Sociología (Zemelman, 1997) concuerdan en que la conciencia y, por ende, el sujeto y sus dimensiones son intersubjetivos y radicalmente sociales $^{3}$. Lo social en esta perspectiva no es una propiedad del sujeto, sino su condición fundamental. Esto quiere decir que ser sujeto es ser en un mundo a la vez histórico y social. Desde esta interpretación, el sujeto se desprende en absoluto de una supuesta estructura fija a priori de la razón o del pensamiento — cogito - y pasa a ser una estructura encarnada, temporal e histórica. Como dice Varela (2000), la conciencia es ahora un asunto público.

Lo público y lo privado dejan de estar en el circuito clásico de interioridad y exterioridad, porque ya no hay más un afuera o adentro absolutos en relación con el sujeto: ambas dimensiones coexisten y están codeterminadas. Esta ambigüedad, fruto de la trama subjetiva de los actores sociales, causa que la tradicional idea de hecho social como algo objetivo que ocurre a los sujetos sea insuficiente para leer la textura de los fenómenos sociales que son fundamentalmente subjetivos o, si se prefiere, intersubjetivos, o sea relacionales, multidimensionales y complejos. Los hechos son interpretaciones $y$, desde este punto de vista, la historia es más bien una hermenéutica que se comprende y no una teoría explicativa de hechos cronológicos. Lo histórico-social no es, por tanto, el resultado de causas y efectos que surgen en la línea de un tiempo y un espacio determinados, sino la condición de la subjetividad humana que se despliega siempre temporalmente desde la experiencia humana y los imaginarios sociales en que esta experiencia se desarrolla y se re-crea de manera constante (Castoriadis, 1986).

Desde esta perspectiva, por ejemplo, el discurso tradicional sobre la indiferencia de los jóvenes y su falta de interés político, fundamentado en las opiniones que manifiestan en las encuestas, ha sido muy cuestionado por las movilizaciones estudiantiles desarrolladas en el año 2011 en Chile.

Frente a la paradoja que caracteriza al sistema democrático chileno, no es extraño que el compromiso ciudadano de los sectores juveniles en

3 Aunque su formación académica corresponde al ámbito de la Biología, la particular visión de lo que Francisco Varela (2000) denomina "conciencia encarnada" le permite reinterpretar la subjetividad en términos de relación radical al mundo y, por ende, a los otros. De esta forma, la afirmación de Varela sobre la conciencia como un "asunto público" implica sostener que lo "público" y el ámbito de lo social no son simplemente una propiedad accidental del ser humano, sino una cualidad de la estructura misma de la conciencia. Este planteamiento, que es sobre todo un argumento, lo discute Varela en distintos ámbitos del conocimiento, desde las ciencias cognitivas hasta la política (Varela \& Hayward, 1997; Castoriadis, 2002). 
la actualidad también presente un carácter paradójico. Los jóvenes siguen manifestando una profunda crítica y desconfianza hacia la institucionalidad y los sectores políticos, pero al mismo tiempo han propiciado una activa participación ciudadana de carácter político, lo que permitiría inferir que su aversión hacia la política responde más bien a la política partidaria y no a la política en su sentido amplio, es decir, como todo aquello referido a la convivencia social y a la esfera comunitaria de la sociedad (Espinoza \& Madrid, 2010; Martínez, Silva \& Hernández, 2010; Zarzuri, 2010).

Lo incongruente de esta situación se acentúa cuando se considera que, para la mayoría de los jóvenes, la ciudadanía está referida al cumplimiento de las leyes (47,1\%); solo en segundo lugar, a la participación activa en la comunidad $(28,7 \%)$ y a la participación en la toma de decisiones $(27,8 \%)$ como tercera opción, pese a que, en la práctica, esta dimensión es la que predomina.

Gráfico 4.

¿Qué es la ciudadanía?

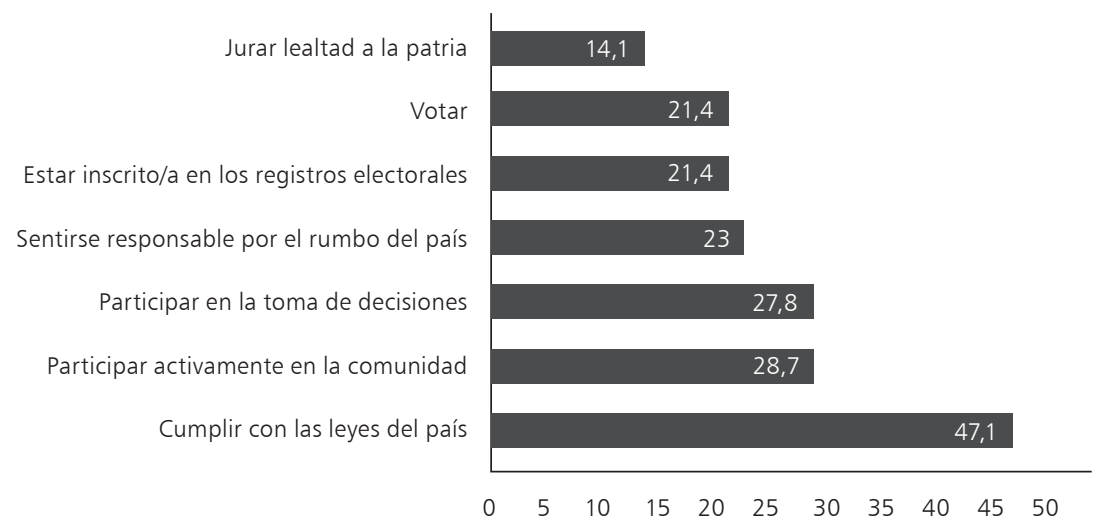

Fuente: Injuv (2010)

La forma en que los jóvenes están participando por medio del movimiento estudiantil ${ }^{4}$ no ha sido un evento aislado, ya que han cuestionado la estructura de la democracia que impera en Chile, debido a las paradojas sistémicas que comprometen al conjunto del orden social y, sobre todo, a las formas estructurales de instalación de un sistema "hiperprivado" de la relaciones sociales y de los derechos fundamentales de las personas. Su impacto en el debate y en la agenda no se ha limitado a los temas educacionales, sino que le ha dado un nuevo impulso a las problemáticas referidas a la estructura del poder legislativo (reforma al sistema binominal) y ha cuestionado la exclusividad de las instituciones representativas en la toma de decisiones, al levantar la propuesta de un plebiscito ciudadano para dar solución a las demandas estudiantiles. Tal ha sido el efecto de

4 El movimiento estudiantil de 2011, que sigue centrado en las mismas reivindicaciones de años anteriores, tiene una historia. La denominada Revolución pingüina (2007) es uno sus antecedentes más cercanos, aunque tuvo su apogeo en estudiantes de enseñanza media, a diferencia del movimiento de 2011, que estuvo focalizado en estudiantes universitarios.

El eje de la reivindicación consignó la necesidad de una educación gratuita y de calidad, en el contexto del sistema económico chileno, ultraliberal, sustentado en una economía social de mercado. Si la educación es concebida como un bien de consumo, es evidente que la reivindicación de los estudiantes apuntó a la recuperación del espacio público para la educación con la finalidad de democratizar el conocimiento en una sociedad como la chilena, hipersegmentada socialmente (Garcés, 2012). 
este movimiento, que incluso para algunos sectores, es el inicio de una nueva etapa que llevará a modificar la estructura del sistema hacia una democracia más directa y participativa (Salazar, 2011).

¿Cómo explicar esta paradoja de la participación juvenil? ¿Por qué el sistema democrático está cada vez más desprestigiado y debilitado, si se observa a una juventud participativa y comprometida con el cambio social? ¿Qué tipo de ciudadanía se está formando para que los jóvenes estén exigiendo un cambio del sistema democrático? Las razones que permiten comprender las paradojas de la ciudadanía juvenil son amplias y encuentran gran parte de su explicación en las propias características del sistema democrático. No obstante, hay una dimensión particular que exige ser considerada por la importancia que se le ha asignado desde el retorno a la democracia: la educación ciudadana en la escuela.

\section{La educación ciudadana en Chile}

La misión de formar a los ciudadanos en la escuela se remonta a la consolidación de los Estados nacionales en las primeras décadas del siglo XIX. El objetivo de esta tarea era la formación de una identidad nacional única y compartida por toda la población, incluidas las comunidades indígenas y los inmigrantes, como requisito básico para convertirse en ciudadanos (Lewkowicz, 2008). Ciudadanía e identidad se transformaron en sinónimos y la enseñanza de la historia patria, asumida como "historia oficial", se convirtió en la base de la formación ciudadana.

A lo largo del siglo XX, la educación ciudadana configuró la asignatura de Educación Cívica, cuya finalidad era promover la formación de ciudadanos comprometidos con el desarrollo político y económico de la Nación por medio de la transmisión de contenidos vinculados a la institucionalidad política y jurídica y a los valores propios de la comunidad nacional (República de Chile, Ministerio de Educación, 2007).

Tras el retorno a la democracia, la reforma curricular impulsada en 1997 enfocó la formación de los ciudadanos hacia una "educación ciudadana", inspirada en los valores y las prácticas democráticas y orientada a formar "ciudadanos activos" que, además de dominar contenidos conceptuales, desarrollaran habilidades y actitudes necesarias para participar activamente en el sistema democrático y de manera comprometida con la sociedad (Cox, 2006; Cox \& García Huidobro, 1999). Para lograr estos objetivos, se vinculó la formación ciudadana a la asignatura de Historia y Ciencias Sociales, transformada en su columna vertebral (República de Chile, Ministerio de Educación, 2007). Esta decisión se justificó en el compromiso ético de esta asignatura con la democracia y los derechos humanos, requisitos fundamentales para desarrollar los conocimientos, las habilidades y las actitudes propias de una sociedad democrática (República de Chile, Ministerio de Educación, 2010). Por esta razón, la educación ciudadana se incorporó de manera transversal al currículo de esta asignatura, desde $1^{\circ}$ Básico a $4^{\circ}$ Medio. Como complemento, algunos aspectos ciudadanos fueron vinculados a los contenidos de otras tres asignaturas: Lenguaje y Comunicación, Filosofía y Orientación y al resto del currículo por medio de los Objetivos Fundamentales Transversales (OFT), dirigidos al desarrollo de habilidades y actitudes que potencien las competencias ciudadanas. No obstante, estas relaciones tienen un carácter menos sistemático, por lo que no se puede hablar de una incorporación generalizada en el currículo, como sí ocurre en otros países de América Latina (Cox, Jaramillo \& Reimers, 2005). Este modelo curricular significó que, a partir de 1997, la tarea de formar a los ciudadanos democráticos dentro de la escuela quedó en manos de los profesores de Historia.

Tras quince años de la puesta en marcha de este nuevo currículo, se puede inferir que no se han obtenido los resultados esperados. A la evidente crisis de representación democrática que está experimentando Chile y que se hace mucho más patente en las generaciones jóvenes, se suma la evolución de los resultados obtenidos en las evaluaciones sobre Educación Cívica y Ciudadanía, aplicadas por la International Association for the Evaluation of Educational Achievement (IEA) en 1999 (Civic Education Study, Cived) y en 2009 (Internacional Civic Education and Citizenship Study, ICCS). Al comparar los resultados de estos estudios (Shulz et al., 2010; Torney-Purta, et al., 2001), se observa que no existen variaciones en relación con el conocimiento y las actitudes ciudadanas. En una década, los estudiantes han mantenido el mismo nivel de conocimientos cívicos, que se ubican bajo el promedio internacional en ambas evaluaciones.

Tabla 2.

Resultados de estudios internacionales sobre educación ciudadana

\begin{tabular}{ll}
\hline \multicolumn{1}{c}{ Cived (1999) } & \multicolumn{1}{c}{ ICCS(2009) } \\
\hline 89 puntos & 89 puntos (483) \\
Bajo promerio Internacional & Bajo promedio internacional \\
\hline \hline
\end{tabular}

Fuente: Cox (2010)

Respecto al compromiso cívico medido a partir de cuatro dimensiones - ciudadanía convencional, ciudadanía social, participación política y confianza en la participación en la escuela-, ambas evaluaciones indican que los jóvenes chilenos se encuentran cerca del promedio internacional, sobre todo en lo que se refiere a la participación en organizaciones sociales, 
aunque se evidencia que esta participación no tiene intencionalidad política y es de carácter individual, como lo han reafirmado las encuestas del Injuv (2006; 2010). Además, solo un 46\% de los encuestados manifestó interés por la política y la mayoría expresó que confiaba más en los medios de comunicación que en las instituciones gubernamentales.

La particularidad de estos resultados es que los estudiantes participantes en el año 2009 han sido formados con el nuevo currículo, mientras que los alumnos que se presentaron en 1999 habían sido formados con el de la dictadura.

Entre las razones que permiten explicar esta situación se deben considerar las dificultades que ha experimentado la implementación del nuevo programa de formación ciudadana. En primer lugar, porque el currículo ha continuado enfatizando la dimensión política de la ciudadanía (Cox, Lira \& Gazmuri, 2009), con contenidos centrados en la institucionalidad política y en el incentivo de una participación responsable y representativa y ha dejado en un segundo plano las dimensiones sociales y civiles de la ciudadanía y el desarrollo de la capacidad crítica que requiere la participación activa (Cox, 2006). En segundo lugar, porque la reforma curricular se hizo sin la participación de los profesores (García Huidobro, 2006), sin considerar que ellos son los encargados de llevarla a la práctica y que la forma como se apropian de las disposiciones curriculares es fundamental para que estas lleguen a los estudiantes (Kerr, 2002). No se recibió su opinión sobre la función formadora de ciudadanía que se les encomendaba, sobre las características del currículo que se les pedía aplicar ni se tuvo en cuenta su grado de preparación para hacerse cargo de esta tarea.

El año 2004, en atención a las dificultades observadas y al creciente desinterés de las generaciones más jóvenes por participar del sistema político electoral, el Ministerio de Educación creó la Comisión de Formación Ciudadana, cuya misión es elaborar sugerencias para mejorar la implementación curricular. Las conclusiones de esta Comisión indicaron que el programa de formación ciudadana no lograba crear una comunidad nacional cohesionada, que sentara las bases de un sistema democrático fuerte y que estimulara el compromiso ciudadano de los jóvenes con la comunidad y la democracia. Las principales razones de esta falla eran, por una parte, la carencia de experiencias escolares que promovieran la integración y la instauración de lazos sociales y, por otro lado, la falta de alineación de las prácticas pedagógicas con los objetivos curriculares (Peña, 2005). Para superar estas dificultades, la Comisión hizo una serie de observaciones orientadas a reorganizar los contenidos curriculares y proponer modelos de prácticas pedagógicas que fortalecieran el quehacer docente con miras a los objetivos curriculares (República de Chile, Ministerio de Educación, 2007). Además, volvió a enfatizar la importancia de la enseñanza de la Historia para la formación ciudadana y la ratificó como el eje en torno al cual se debía formar a la ciudadanía.

El crítico diagnóstico que existe sobre la falta de alineación entre el currículo y las prácticas pedagógicas no es una situación exclusiva de Chile, sino que es una de las principales características de la educación ciudadana en el mundo, tal como lo ha mostrado la investigación reciente (Clark, 1999; Cerda et al., 2004; laies \& Delish, 2009; Kerr, 2002; Lewskovicz, 2008; Pagès \& Santiesteban, 2006; Tedesco, 1995). La formación de ciudadanos activos y democráticos, dotados de habilidades y competencias para enfrentar los desafíos de la globalización, se está implementando con estrategias pedagógicas tradicionales, centradas en la exposición del profesor y en la transmisión de contenidos, sin espacios suficientes para que los alumnos construyan activamente su conocimiento. Más allá de este 
diagnóstico, cabe preguntarse hasta qué punto la educación es capaz de suplir las carencias que el propio sistema genera o, al menos, contribuir a la formación ciudadana que el actual sistema requiere.

\section{El efecto de la escuela en la formación ciudadana}

La escuela no es la única instancia formadora de ciudadanía; es más, la mayoría de las veces son mucho más influyentes la familia, la comunidad, los medios de comunicación y las redes sociales (Green, 2006; Reimers, 2006). No obstante, la escuela tiene la ventaja de propiciar un espacio social que permite medir sus efectos sobre el compromiso ciudadano (Reimers, 2006). Durante la última década, se ha desarrollado una serie de investigaciones orientadas a medir este efecto (Callahan, Muller \& Schiller, 2010; Dobozy, 2007; Kahne \& Sporte, 2008; Quintelier, 2010), pero no ha arrojado conclusiones definitivas sobre el real impacto que tiene la educación escolar, porque los resultados presentan diferencias importantes en la significación del efecto y sus mediciones han estado orientadas en exclusiva al compromiso ciudadano en un sentido político electoral y han dejado de lado las dimensiones cívica, social y cultural de la ciudadanía. Lo que estas conclusiones sí han permitido establecer son los factores escolares más influyentes en la formación ciudadana: las experiencias escolares, las oportunidades curriculares y las experiencias pedagógicas.

Las experiencias escolares corresponden al clima escolar y a los espacios de participación y expresión que se generan dentro de la escuela. En esta área, las investigaciones coinciden en que existe una correlación positiva entre el compromiso ciudadano y un clima escolar que no solo estimule la participación activa de los estudiantes, sino que también ofrezca espacios para la expresión y la deliberación (Callahan, Muller \& Schiller, 2010; Dobozy, 2007).

El currículo, por su parte, es un elemento clave dentro de cualquier sistema educativo, porque establece las oportunidades de aprendizaje mediante una selección cultural que organiza la trayectoria y la experiencia escolar (Bernstein, 1998). Esta selección define los objetivos y los contenidos en atención a la visión que la sociedad tiene de sí misma y que busca transmitir a las nuevas generaciones (Kerr, 2002; Faulks, 2006). Para las sociedades modernas, diversas por definición, esta visión de la sociedad no es homogénea y debe compatibilizar las distintas concepciones de ciudadanía y las múltiples demandas sociales y culturales que conviven dentro de ella, lo que muchas veces deriva en la desarticulación entre los objetivos y los contenidos propuestos por el mismo currículum. Los estudios comparativos que han analizado los currículos de formación ciudadana en distintos países (Cox, 2006, 2010; Cox, Lira \& Gazmuri, 2009; Davies \& Issit, 2005; Kerr, 2002; Osler \& Starkey, 2001; Suárez, 2008) han identificado que, tanto en el mundo anglosajón como en América Latina, los programas curriculares de formación ciudadana buscan desarrollar una ciudadanía activa, con énfasis en el respeto al otro y a la diversidad, pero sin que esto implique renunciar al objetivo de lograr la homogenización identitaria de los ciudadanos e inculcar formas de participación únicas, con alusión indirecta o superficial al multiculturalismo. Así mismo, las investigaciones centradas en la implementación de los currículos ciudadanos evidencian falencias importantes por la falta de coherencia entre los objetivos del currículo, sus contenidos y las prácticas pedagógicas propuestas para su implementación (Macintyre \& Simpson, 2009; Westheimer \& Kahne, 2004).

Las experiencias pedagógicas hacen referencia al proceso educativo desarrollado por los docentes. Considerar el rol que desempeñan los 
profesores en la educación ciudadana es fundamental, no solo porque sus capacidades y la calidad de sus prácticas se han convertido en el factor más decisivo para el logro general de los aprendizajes (Barber \& Mourshed, 2008; Luyten, Visscher \& Witziers, 2005; Stevenson \& Stiegler, 1999), sino porque son los encargados de llevar a la práctica las disposiciones curriculares. Sin embargo, es un área de estudio poco desarrollada en el mundo y, en particular, en Chile.

Las investigaciones han identificado la poca preparación de los profesores para implementar los programas de educación ciudadana (Oulton, Day, Dillon \& Grace, 2004; Schweisfurth, 2006; Torney Purta, Barber \& Klandl, 2005). La mayoría de ellos tiene una especialización en Historia o Ciencias Sociales, pero no en ciudadanía, por lo que no cuenta con las herramientas conceptuales y pedagógicas necesarias para asumir las exigencias curriculares. Además, han identificado que las convicciones y creencias a partir de las cuales interpretan el currículo influyen de modo directo en la forma de implementarlo, pese a que existe poca coherencia entre la forma en que conciben la ciudadanía y sus estrategias pedagógicas (Alviar, Randall, Usher \& Engelhard, 2008; Cerda, Egaña, Magendzo, Santa Cruz \& Vargas, 2004; Down, Ditchburn \& Lee, 2008; Kerr, 2002; Marri, 2005; Martínez, Urquijo, Valls, Yague \& Crespo, 2008; Torney Purta, Barber \& $\mathrm{Klandl}$, 2005). Buena parte de estas investigaciones no apunta a comprender las creencias y convicciones de los profesores en sí mismas como dimensiones fundamentales en la toma de decisiones que inspiran el quehacer docente, sino que están relacionadas con su efecto en los aprendizajes de los alumnos o con el tipo de prácticas que desarrollan. Entre las excepciones se pueden citar los estudios de Sim (2008), Peterson (2009) y Tutiaux-Guillon (2003), que buscan determinar las concepciones ciudadanas de los profesores; los trabajos de Klein (2010) y Schweisfurth (2006), que apuntan a comprender cómo se apropian los profesores de las exigencias curriculares y las investigaciones de Cunningham (2009), Maggioni, Van Sledright y Alexander (2009) y Van Sledright (2005), que indagan sobre las concepciones epistemológicas de los docentes. Los aportes de estas investigaciones han sido clave para comprender la forma en que las concepciones de los profesores determinan el desarrollo de las prácticas pedagógicas, pero solo entregan visiones parciales, porque las dimensiones ciudadanas, curriculares y epistemológicas actúan de manera simultánea en la toma de decisiones (Martínez, Urquijo, Valls, Yague \& Crespo, 2008).

Junto a estos elementos fundamentales en el proceso educativo, es indispensable comprender de qué manera deben organizarse los elementos curriculares y pedagógicos para contribuir a la formación de la ciudadanía democrática. En las últimas décadas, se ha elaborado una serie de clasificaciones sobre los tipos de educación ciudadana existentes (Cellier, 2003; Cornbleth, 1982; Galichet, 1998; 1999; Mougniotte, 1999; Westheimer \& Kahne, 2000, citados en Pagés \& Santiesteban, 2006). Entre ellas, el modelo ofrecido por David Kerr (2002) sintetiza las visiones interpretativas a partir de las cuales se construyen los modelos educativos: la minimalista y la maximalista. La interpretación minimalista se fundamenta en una ciudadanía concebida como estatus y ha dado origen a la educación cívica o educación sobre la ciudadanía, que busca promover una identidad única mediante modelos educativos tradicionales, centrados en la transmisión de conocimientos vinculados a la historia nacional y al funcionamiento político y jurídico de las instituciones. Las estrategias didácticas implementadas se organizan a partir del rol protagónico del profesor, mientras que la participación de los estudiantes es limitada.

La interpretación maximalista se fundamenta en una ciudadanía concebida como acción y ha dado origen a la educación ciudadana. El modelo educativo está orientado a potenciar habilidades y competencias básicas para una ciudadanía participativa, por medio de una pedagogía crítica e interactiva, que promueve estrategias de deliberación y debate, el service learning y la participación democrática dentro del espacio escolar. Dentro de estas interpretaciones maximalistas, se pueden reconocer dos orientaciones (Kerr, 2002). La primera, denominada educación mediante la ciudadanía, está enfocada al desarrollo de la ciudadanía activa o comunitaria, para estimular el desarrollo de la identidad nacional y de las dimensiones política, civil y social de la ciudadanía. Se ha destacado por ser la propuesta inspiradora de los programas de formación ciudadana aplicados desde la década del noventa en Inglaterra, Australia, Estados Unidos, Canadá y Chile (Cox, 2006; Davies \& Issitt, 2005; Faulks, 2006; Olssen, 2004; Osler \& Starkey, 2001). La segunda orientación, denominada educación para la ciudadanía, busca integrar a las otras dos aproximaciones para desarrollar una educación multicultural o global que incorpore las cuatro dimensiones ciudadanas (Banks, 2008), que promueva el desarrollo de identidades escalonadas (comunitaria, local, nacional y global) y que estimule el sentido de comunidad y el compromiso que se requieren para sostener la estabilidad del sistema democrático (Marshall, 2009; Osler \& Starkey, 2003).

Esta educación para la ciudadanía, al sintetizar tanto la educación cívica como la ciudadana, ofrece la aproximación más adecuada para desarrollar la cohesión social que exigen los desafíos de la globalización. La educación ciudadana debe estar orientada a formar un capital social amplio, al fortalecer los vínculos intragrupos que se forman dentro de la comunidad de los 
iguales (capital social vinculante) y las relaciones entre grupos (capital puente), que se forman entre los miembros de distintas comunidades (Putnam, 2000). Con el desarrollo de estos dos tipos de vínculos se estimularía el desarrollo de la identidad étnica, local, regional, nacional, etc., sin descuidar el sentido de pertenencia necesario para comprometerse con el sistema democrático ni la tolerancia y confianza necesarias para generar acciones colectivas entre miembros de distintas comunidades (Granovetter, 1973; Putnam, 2000). Esto implica superar la visión peyorativa en relación con la educación cívica, fundamental para el impulso del capital social vinculante, mientras que la educación ciudadana deberá estar orientada al desarrollo del capital social puente (Cox, Lira \& Gazmuri, 2009), preciso para la convivencia pacífica dentro de las sociedades multiculturales y complejas de la actualidad. Por este motivo, identificar el concepto de ciudadanía y la visión interpretativa desde donde se construye el programa de formación ciudadana, es clave para comprender de qué forma está contribuyendo a la cohesión social y al fortalecimiento del sistema democrático.

\section{Consideraciones finales}

Desde Hegel y Marx, las paradojas y contradicciones sociales han sido interpretadas como el motor de la historia. En presencia de otros diagnósticos (Fukujama, 2002; Lipovestky, 1986), las sociedades se han homogenizado y las reivindicaciones y los conflictos se sostienen local y regionalmente. La historia parece agitarse más por "causas", en el sentido de motivos contingentes, que por grandes ideas que movilicen a la sociedad en su conjunto.

Por esta razón, la idea de una paradoja fundacional relativa al sistema democrático permite constatar los límites inherentes a todo sistema y detectar las estructuras que fragilizan la instauración interna del sistema democrático. Este sistema, según la tendencia actual en nuestra cultura occidental, se presenta permeabilizado por un modelo económico ultraliberal que, además de promover un concepto del individuo y de sociedad sustentada solo en el producto y la ganancia de las cosas, genera una atomización radical del sujeto y del espacio público, tras alterar el sentido profundo de la cohesión social.

La desconfianza en las instituciones es transversal tanto en los jóvenes como en los adultos; el déficit de la educación ciudadana no es una cuestión del currículo ni de los límites inherentes a la escuela. El sistema educativo es el mejor ejemplo de un "sistema" que no es más un sistema en el sentido de ser una entidad abierta, comunicante, emergente, donde el todo sea cualitativamente mayor a la suma de las partes. Un sistema como el educativo, que por defecto segrega y reproduce la inequidad de sí mismo no es, en rigor, un sistema. La raíz de la paradoja de la experiencia ciudadana en los jóvenes se anida en esta desestructura que, de una parte, tiende a romper los lazos de la subjetividad social de los individuos y a debilitar aún más la cohesión social y, de la otra, fortalece el sentimiento compartido de abrir un nuevo espacio para la utopía, la comunidad y las ideas.

\section{Sobre los autores}

Luis Manuel Flores-González es doctor en Filosofía. Profesor titular de la Pontificia Universidad Católica de Chile, responsable del área de Epistemología de la Educación del Programa de Doctorado en Ciencias de la Educación y profesor de la cátedra de Epistemología de las Ciencias Biológicas. Miembro del Comité Científico del Instituto internacional de política y civilización, Poitiers (Francia).

Carolina Andrea García-González es licenciada en Historia y Educación, magíster en Ciencias de la Educación y doctora en la misma área. Sus intereses se orientan a la Epistemología de la Historia y la Didáctica de la Ciencias Sociales y a la investigación que integra la enseñanza de la historia con la formación ciudadana.

\section{Referencias}

Alviar, T., Randall, J. D., Usher, E. L., \& Engelhard Jr., G. (2008). Teaching Civic Topics in Four Societies: Examining National Context and Teacher Confidence. Journal of Educational Research, 101 (3), 177-187.

Arendt, H. (2003). La condición humana. Buenos Aires: Paidós.

Baeza J. (2012). Vinculaciones entre la construcción y deconstrucción de la confianza y la cohesión social, en jóvenes estudiantes de educación secundaria: lineamientos para fortalecer la democracia. (Investigación inédita). Proyecto Fondecyt, Santiago.

Banks, J. A. (2008). Diversity, Group Identity, and Citizenship Education in a Global Age. Educational Researcher, 37 (3), 129-139.

Barber, M., \& Moursehd, M. (2008). Cómo hicieron los sistemas educativos con mejor desempeño del mundo para alcanzar sus objetivos. Santiago: Preal.

Bernstein, B. (1998). Conocimiento oficial e identidades pedagógicas: la política de recontextualización. En Pedagogía, control simbólico e identidad. Barcelona: Morata.

Callahan, R. M., Muller, C., \& Schiller, K. S. (2010). Preparing the Next Generation for Electoral Engagement: Social Studies and the School Context. American Journal of Education, 116 (4), 525-556. 
Castoriadis, C. (1986). El campo de lo social histórico. Estudios, (4), 7-26.

Castoriadis, C. (2002). La insignificancia y la imaginación. Diálogos con Daniel Mermet, Octavio Paz, Francisco Varela. Madrid: Mínima Trotta.

Cavallo, A., Salazar, M., \& Sepúlveda, O. (2004). La historia oculta del régimen militar: 1973-1990. Santiago: De Bolsillo.

Centro de Estudios de la Realidad Contemporánea [CERC]. (2011). Barómetro de la Política. Agosto-septiembre. Recuperado de http://www. cerc.cl/cph_upl/20121029112544_barometro_de_la_politica_agosto-septiembre2011.pdf

Cerda, A. M., Egaña, M. L., Magendzo, A., Santa Cruz, E., \& Varas, R. (2004). El complejo camino de la formación ciudadana. Santiago: LOM-PIIE.

Clark, T. (1999). Rethinking Civic Education for the 21st Century. En D. Marsh, Preparing Our Schools for the 21st Century. ASCD Yearbook. Alexandria: ASCD Yearbook.

Cornejo, R., González, J., \& Caldichoury, J. P. (2007). Participación e incidencia en las políticas educativas: el caso chileno. Buenos Aires: Flape.

Cox, C. (2006). Jóvenes y ciudadanía política en América Latina. Desafíos al currículo. Prelac, (3), 64-73.

Cox, C. (2010). Oportunidades de aprendizaje escolar de la ciudadanía en América Latina: currículos comparados. Bogotá: SREDECC.

Cox, C., \& García Huidobro, J. E. (1999). La reforma educacional chilena 1990-1998. Visión de conjunto. En J. E. Huidobro (ed.). La reforma educacional chilena. Santiago: Popular.

Cox, C., Jaramillo, R., \& Reimers, F. (2005). Educar para la ciudadanía y la democracia en las Américas: una agenda para la acción. Washington: Banco Interamericano de Desarrollo.

Cox, C., Lira, R., \& Gazmuri, R. (2009). Currículos escolares y sus orientaciones sobre historia, sociedad y política: significados para la cohesión social en Latinoamérica. En S. Schwartzman \& C. Cox (eds.). Políticas educativas y cohesión social en América Latina. Santiago: Uqbar.

Cunningham, D. L. (2009). An Empirical Framework for Understanding how Teachers Conceptualize and Cultivate Historical Empathy in Students. Journal of Curriculum Studies, 41 (5), 679-709.

Davies, I., \& Issitt, J. (2005). Reflections on Citizenship Education in Australia, Canada and England. Comparative Education, 41 (4), 389-410.

Dobozy, E. (2007). Effective Learning of Civic Skills: Democratic Schools Succeed in Nurturing the Critical Capacities of Students. Educational Studies, 33 (2), 115-128.

Down, B., Ditchburn, G., \& Lee, L. (2008). Teacher's Ideological Discourses and the Enactment of Citizenship Education. Curriculum Perspectives, 28 (2), 1-27.

Espinoza, V., \& Madrid, S. (2010). Trayectoria y eficacia política de los militantes en juventudes políticas. Estudio de la elite política emergente. Santiago: Intituto de Estudios Avanzados Universidad de Santiago de Chile.

Faulks, K. (2006). Education for Citizenship in England's Secondary Schools: A Critique of Current Principle and Practice. Journal of Education Policy, 21 (1), 59-74.

Fukuyama, F. (1992). El fin de la historia y el último hombre. Buenos Aires: Planeta.

Garcés, M. (2012). El despertar de la sociedad. Los movimientos sociales en América Latina. Santiago: LOM.

García Huidobro, J. E. (2006). Conflictos y alianzas en las reformas educativas. Siete tesis basadas en la experiencia chilena. En S. Martinic 
\& M. Pardo (eds.). Economía política de las reformas educativas en América Latina. Santiago: CIDE, Preal.

García, C. (2006). El peso de la memoria en los inicios de la transición a la democracia en Chile 19871989. Historia, 39 (2), 431-275.

Girard, R. (1982). El misterio de nuestro mundo. Salamanca: Sígueme.

Girard, R. (2009). La violencia y lo sagrado. Barcelona: Anagrama.

Granovetter, M. (1973). The Strenght of Weak Ties. The American Journal of Sociology, 78 (6), 1360-1380.

Green, A. (2006). Education, Globalization and the Nation State. En H. Lauder, P. Brown, J. Dillabough \& A. H. Halsey (eds.). Education, Globalization, and Social Change. Oxford: Oxford University Press.

Huneeus, C. (2000). El régimen de Pinochet. Santiago: Sudamericana.

laies, G., \& Delich, A. (2009). Sistemas educativos y cohesión social: la reconstrucción de "lo común" en los Estados nacionales del siglo XXI. En S. Schwartzman \& C. Cox (eds.). Políticas educativas y cohesión social en América Latina. Santiago: Uqbar.

Instituto Nacional de la Juventud [Injuv]. (2006). V Encuesta Nacional de la Juventud. Santiago: Autor.

Instituto Nacional de la Juventud [Injuv]. (2010). VI Encuesta Nacional de la Juventud. Santiago: Autor.

Kahne, J. E., \& Sporte, S. E. (2008). Developing Citizens: The Impact of Civic Learning Opportunities on Students' Commitment to Civic Participation. American Educational Research Journal, 45 (3), 738-766.

Kerr, D. (2002). Citizenship Education in the Curriculum: An International Review. The School Field, $X(3-4), 5-32$.

Klein, S. (2010). Teaching History in the Netherlands: Teachers' Experiences of a Plurality of Perspectives. Curriculum Inquiry, 40 (5), 614-634.

Lechner, N. (2002). Las sombras del mañana. La dimensión subjetiva de la política. Santiago: LOM Ediciones.

León, E., \& Zemelman, H. (coords.) (1997). Subjetividad: umbrales del pensamiento social. Barcelona: Anthropos.

Lewkowicz, I. (2008). Pedagogía del aburrido. Buenos Aires: Paidós.

Lipovetsky, G. (1986). La era del vacío: ensayos sobre el individualismo contemporáneo. Madrid: Anagrama.

Luyten, H., Visscher, A., \& Witziers, B. (2005). Scholl Effectiveness Research: From a Review of the Criticism to Recommendation for Further Development. School Effectiveness and Scholl Improvement, 16 (3), 249-279.
Macintyre, S., \& Simpson, N. (2009). Consensus and Division in Australian Citizenship Education. Citizenship Studies, 13 (2), 121-134.

Maggioni, L., VanSledright, B., \& Alexander, P. A. (2009). Walking on the Borders: A Measure of Epistemic Cognition in History. Journal of Experimental Education, 77 (3), 187-214.

Marri, A. R. (2005). Building a Framework for Classroom-Based Multicultural Democratic Education: Learning from Three Skilled Teachers. Teachers College Record, 107 (5), 1036-1059.

Marshall, H. (2009). Educating the European Citizen in the Global Age: Engaging with the Post-National and Identifying a Research Agenda. Journal of Curriculum Studies, 41 (2), 247-267.

Martínez, L., Silva, C., \& Hernández, A. (2010). ¿En qué ciudadanía creen los jóvenes? creencias, aspiraciones de ciudadanía y motivaciones para la participación sociopolítica. Psykhe, 19 (2), 27-35.

Martínez, N., Urquijo, J., Valls, R., Yague, M., \& Crespo, J. C. (2008). El desarrollo del currículo de Historia en Bachillerato y el uso de los textos: la visión del profesorado. En R. M. Ávila Ruiz, M. A. Cruz Rodríguez \& M. C. Díez Bedmar (eds.). La didáctica de las Ciencias Sociales en los nuevos planes de estudio. Jaén: Universidad de Jaén.

Olssen, M. (2004). From the Crick Report to the Parekh Report: Multiculturalism, Cultural Difference, and Democracy - The Re-Visioning Of Citizenship Education. British Journal of Sociology of Education, 25 (2), 179-192.

Osler, A., \& Starkey, H. (2001). Citizenship Education and National Identities in France and England: Inclusive or Exclusive? Oxford Review of Education, 27 (2), 287-305.

Osler, A., \& Starkey. (2003). Learning for Cosmopolitan Citizenship: Theoretical Debates and Young People's Experiences. Educational Review, 55 (3), 243-254.

Otano, Rafael (1995). Crónica de la transición. Santiago: Planeta.

Oulton, C., Day, V., Dillon, J., \& Grace, M. (2004). Controversial Issues-Teachers' Attitudes and Practices in the Context of Citizenship Education. Oxford Review of Education, 30 (4), 489-507.

Pagès, J. (2003). Ciudadanía y enseñanza de la historia. Revista de la APEHUN, (1), 11-42.

Pagès, J., \& Santisteban, A. (2006). La educación para la ciudadanía hoy. Barcelona: Wolters Kluwer.

Peña, C. (2005). Presentación propuesta Comisión de Formación Ciudadana. Santiago: Ministerio de Educación.

Peterson, A., \& Knowles, C. (2009). Active Citizenship: A Preliminary Study into Student Teacher Understandings. Educational Research, 51 (1), 39-59. 
Programa de las Naciones Unidas para el Desarrollo [PNUD]. (2002). Desarrollo humano en Chile: nosotros los chilenos: un desafío cultural. Santiago: LOM Ediciones.

Programa de las Naciones Unidas para el Desarrollo [PNUD]. (2004). La democracia en América Latina: hacia una democracia de ciudadanos y ciudadanas. Buenos Aires: Aguilar.

Putnam, R. D., Cohen, D., \& Feldstein, L. M. (2000). Bowling Alone: The Collapse and Revival of American Community. Nueva York: Simon \& Shuster.

Quintelier, E. (2010). The Effect of Schools on Political Participation: A Multilevel Logistic Analysis. Research Papers in Education, 25 (2), 137-154.

Reimers, F. (2006). Citizenship, Identity and Education: Examining the Public Purposes of School in an Age of Globalization. Prospects, XXXVI (3), $275-294$.

República de Chile, Ministerio de Educación. (1998). Objetivos fundamentales y contenidos mínimos obligatorios de la Educación Media. Santiago: Autor.

República de Chile, Ministerio de Educación. (2007). Programa de Formación Ciudadana. Santiago: Autor.

República de Chile, Ministerio de Educación. (2010). Ajuste curricular. Fundamentos del programa de estudio Historia y Geografía $1^{\circ}$ Medio. Santiago: Mineduc.

Riquelme, A. (1999). ¿Quiénes y por qué "no están ni ahí"? Marginación y automarginación en la democracia transicional. Chile 1988-1997. En P. Drake \& I. Jaksic (eds.). El modelo chileno. Santiago: LOM.

Salazar, G. (2011). En el nombre del poder popular constituyente. Santiago: LOM.

Schweisfurth, M. (2006). Education for Global Citizenship: Teacher Agency and Curricular Structure in Ontario Schools. Educational Review, 58 (1), 41-50.

Servicio Electoral. (2010). Padrón electoral. Grupos etarios y sexo, en cantidades y porcentajes 1988-2009. Recuperado de http://www.servel. $\mathrm{cl} /$ servlet/Satellite?c=Page\&cid=1374098176919\&pagename=Serve IOficial\%2FPage\%2FSO Padron-ResumenHistorico

Shulz, W., Ainley, J., Fraillo, J., Kerr, D., \& Losito, B. (2010). Initial Findings from the IEA International civis and Citizenship Education Study. Amsterdam: IEA.

Sim, J. B. Y. (2008). What does Citizenship Mean? Social Studies Teachers' Understandings of Citizenship in Singapore Schools. Educational Review, 60 (3), 253-266.

Stevenson, H., \& Stiegler, J. (1999). ¿Por qué los escolares de Asia Oriental tienen alto rendimiento académico? Estudios Públicos, (76) 297-357.

Suárez, D. (2008). Rewriting Citizenship? Civic Education in Costa Rica and Argentina. Comparative Education, 44 (4), 485-503.

Tedesco, J. C. (1995). El nuevo pacto educativo. Madrid: Anaya.

Toledo, M. I., \& Gazmuri, R. (2009). Obedientes memoriones o reflexivos pensantes: tensiones entre objetivos identitarios y cognitivos en enseñanza de la historia reciente de Chile en $6^{\circ}$ año de Enseñanza Básica. Estudios Pedagógicos, XXXV (2), 155-172.

Torney-Purta, J., Henry Barber, C., \& Klandl, W. (2005). How Teacher's Preparations Relates to Student's Civic Knowledge and Engagement in the United States. Recuperado de http://www.civicyouth.org/PopUps/FactSheets/FS_IEA_Teacher_prep.pdf 
Torney-Purta, J., Lehmann, R., Oswald, H., \& Shulz, W. (2001). Citizenship and Education in Twenty-eight Countries. Civic Knowledge and Engagement at Age Fourteen. Amsterdam: IEA.

Tutiaux-Guillon, N. (2003). Los fundamentos de una investigación sobre la concepción de las finalidades cívicas y culturales del profesorado de Geografía e Historia. Enseñanza de las Ciencias Sociales, (2), 27-35.

Van Sledright, B. (2005). Reseña del libro History Lessons: Teaching, Learning, and Testing in US History Classrooms, por S. G. Grant. Journal of Curriculum Studies, 37 (3), 366-370.

Varela, F., \& Hayward J. (1997). Un puente para dos miradas. Conversaciones con el Dalai Lama sobre las ciencias de la mente. Santiago: Dolmen.

Westheimer, J., \& Kahne, J. (2004). What Kind of Citizen? The Politics of Educating for Democracy. American Educational Research Journal, 41 (2), 237-269.

Yarza, C. (2005). ¿Ciudadanía postpolítica? El legado liberal y la despolitización. Opción, 21 (47), 138-157.

Zarzuri, R. (2010). Tensiones y desafíos en la participación política juvenil en Chile. Utopía y Praxis Latinoamericana, 15 (50), 103-115. 